\title{
Geodesics in the Kerr-Newman anti de Sitter spacetimes
}

\author{
H. Shanjit ${ }^{1^{*}}$ and K. Yugindro Singh ${ }^{2}$ \\ ${ }^{1}$ Department of Physics, Rajiv Gandhi University, Rono Hills - 791112, Arunachal Pradesh, India \\ ${ }^{2}$ Department of Physics, Manipur University, Imphal - 795003, Manipur, India \\ Email: shanjitheisnam@gmail.com
}

\begin{abstract}
In this work, the geodesic equations of motion of a test particle in the Kerr-Newman $(\mathrm{KN})$ anti de Sitter spacetimes are presented in the light of the Hamiltonian-Jacobi formalism. We have examined, in the frame of KN anti de Sitter spacetimes, the spherical and circular orbits that the test particle may describe in a rotating charged black hole, discarding the gravitational and electromagnetic radiation and found that bounded spherical and circular stable orbits can exist in the regions of $0<r<r_{-}$in which $r_{-}$is the inner event horizon of the black hole.
\end{abstract}

Keywords: Kerr-Newman anti de Sitter spacetimes; geodesic equations; spherical and circular orbits; gravitational and electromagnetic radiation; inner event horizon; black hole.

\section{Introduction}

A Kerr-Newman anti de Sitter black hole is the generalised rotating Kerr black hole which is an axisymmetric solution of the Einstein-Maxwell equations with the cosmological constant $\Lambda$ taken into account[1]. The geometrical structure as the solutions with the cosmological constant $\Lambda$, provides asymptotically de Sitter spacetimes under the consideration of an attractive cosmological constant, $\Lambda<0$. It may contain black hole horizons along with its singularity. It has been shown recently that the anti de Sitter spacetime plays an important role in the multidimensional string theory[2]. The cosmological constant not only plays important roles in higher gravitational physics but also, it has become one of the most fundamental spacetime curvature constants and is likely the most probable candidate that would explain the late acceleration of the universe. It has the potential to solve one of the mysteries of current observations, the so called Dark Energy problem [3], [4], [5]. Additionally, spacetime geometry, galaxy peculiar velocities, structure formation and early universe physics, support a flat universe model with a cosmological constant[6]. Such a scenario has motivated to introduce spherical symmetric spacetimes with cosmological constant for studying the effects of vacuum energy predicted by Einstein. An approach for understanding the physical properties of spacetimes is to investigate the behaviour of a test particle. Chandrasekhar[7] discussed the general types of particle orbits in a black hole gravitational field: orbits of the first kind which are completely confined outside the black hole event horizon, and orbits of the second kind which penetrate inside the black hole. In the case of rotating or charged black hole, there are bound periodic planetary orbits known as the orbits of the third kinds which neither come out nor terminate at the central singularity[8]. The third kind of bound orbits inside the black hole horizon were discussed by [9], [10] for charged particle in the vicinity of the rotating charged black holes and by [11] for neutral particle. The third kind of bound circular orbits that can exist in the KN de/anti de Sitter spacetimes were again discussed by[12] for both neutral and charged particles.

The physical properties of the Kerr-Newman anti de Sitter spacetimes are appropriately described by their geodesic structure which determines the motion of a test particle. The motion of such a test particle in a spherical region can be considered in a relatively simple and easily tractable way. We shall analyse the properties of the circular and spherical motion for the black hole spacetimes with the repulsive cosmological constant. In Kerr-Newman anti de Sitter black hole spacetimes with appropriately tuned parameters, an unusual effect might exist resulting a spherical stable orbit which is bounded in the regions of $0<r<r_{-}$in which $r_{-}$is the inner event horizon of the black hole, by ignoring the gravitational and electromagnetic radiation in the frame of KN anti de Sitter spacetime. No such effect appears in the spherically symmetric Schwarzschild-anti de Sitter and Reissner-Nordstrom-anti de-Sitter spacetimes in which the repulsive barrier always diverges at the black hole and cosmological horizons. 
In this paper, we attempt to investigate the geometrical properties of the Kerr-Newman anti de sitter spacetimes with non zero cosmological constant and to explain as how the Carter's constant affects motion out of the equatorial plane. The properties of spherical orbit of a test particle in the gravitational field of KN anti de Sitter black hole are analysed. We will use the units $G=c=1$ throughout the paper.

\subsection{Kerr-Newman anti de Sitter Geometry}

In the standard Boyer-Lindquist coordinates $(t, r, \theta, \phi)$, the Kerr-Newman anti de Sitter spacetimes interior to the cosmological horizon and exterior to the black hole embedding in the background of the KN anti de sitter spacetimes, is completely determined by the four parameters: the charge $e$, mass $M$, angular momentum $a(=J / M)$ per unit mass of the black hole and non zero cosmological constant, $\Lambda$. The corresponding metric with a non zero cosmological constant takes the form [13]:

$$
d s^{2}=\frac{-\Delta_{r}}{\rho^{2}}\left(\frac{d t}{I}-\frac{a \sin ^{2} \theta d \phi}{I}\right)^{2}+\frac{\Delta_{\theta} \sin ^{2} \theta}{I^{2}}\left[\frac{a d t}{\rho}-\frac{\left(r^{2}+a^{2}\right) d \phi}{\rho}\right]^{2}+\rho^{2}\left(\frac{d r^{2}}{\Delta_{r}}+\frac{d \theta^{2}}{\Delta_{\theta}}\right),
$$

where the functions $\rho, \Delta_{r}, \Delta_{\theta}$ and $I$ are defined respectively by

$$
\begin{aligned}
\rho^{2} & =r^{2}+a^{2} \cos ^{2} \theta \\
\Delta_{r} & =\left(1-\frac{\Lambda r^{2}}{3}\right)\left(r^{2}+a^{2}\right)-2 M r+e^{2}, \\
\Delta_{\theta} & =\left(1+\frac{\Lambda a^{2} \cos ^{2} \theta}{3}\right), \\
I & =1+\frac{\Lambda a^{2}}{3} .
\end{aligned}
$$

The electromagnetic field for the source is given by the vector potential

$$
A_{\mu}=\frac{e r}{I \rho^{2}}\left[\delta_{\mu}^{t}-a \sin ^{2} \theta \delta_{\mu}^{\phi}\right]
$$

We take $a$ to be positive. If $e=0$ then, the electromagnetic field tensor Eq.(6) vanishes and the metric Eq.(1) satisfies the vacuum Einstein's field equations. The corresponding non-zero contravariant components $g^{i j}$ of the metric are obtained as

$$
\begin{aligned}
g^{t t} & =\frac{I^{2}}{\Delta_{r} \Delta_{\theta} \rho^{2}}\left[\Delta_{r} a^{2} \sin ^{2} \theta-\Delta_{\theta}\left(r^{2}+a^{2}\right)^{2}\right], \\
g^{\phi t} & =g^{t \phi}=\frac{I^{2}}{\Delta_{r} \Delta_{\theta} \rho^{2}}\left[\Delta_{r}-\Delta_{\theta}\left(r^{2}+a^{2}\right)^{2}\right], \\
g^{\phi \phi} & =I^{2}\left[\frac{1}{\sin ^{2} \theta \Delta_{\theta} \rho^{2}}-\frac{a^{2}}{\Delta_{r} \rho^{2}}\right], \\
g^{r r} & =\frac{\Delta_{r}}{\rho^{2}} \\
g^{\theta \theta} & =\frac{\Delta_{\theta}}{\rho^{2}} .
\end{aligned}
$$

The quanity $\Lambda$ can be identified as the cosmological constant associated with the characteristic length scale $\ell$ taken as $\frac{-3}{\ell^{2}}$. We can find the horizon radii by finding the roots of the equation $\Delta_{r}=0$. For simplicity we take the roots as $r_{n}, r_{-}, r_{+}$and $r_{c}$, i.e.

$$
\Delta_{r}(r)=\left(r-r_{n}\right)\left(r-r_{-}\right)\left(r-r_{+}\right)\left(r-r_{c}\right),
$$

where $r_{-}$and $r_{+}$are the inner and outer horizons of the black hole respectively. $r_{c}$ is the cosmological horizon for observers between $r_{c}$ and $r_{+} \cdot r_{n}(<0)$ is the another cosmological horizon inside the singularity at $r=0$. The roots are obtained as in[14]:

$$
r_{ \pm}=X_{+} \pm \ell \sqrt{Y_{-}}, r_{n}=r_{c}^{*}=-X_{+}+i \ell \sqrt{Y_{+}},
$$


where

$$
X_{+}=\sqrt{\frac{\left(\ell^{2}+a^{2}\right)}{6}}\left[\sqrt{1+\frac{12 \ell^{2}\left(a^{2}+e^{2}\right)}{\left(\ell^{2}+a^{2}\right)^{2}}} \cosh \left(\frac{1}{3} \cosh ^{-1} \frac{\left(4+\frac{54 M^{2} \ell^{4}}{\left(\ell^{2}+a^{2}\right)^{3}}-3\left(1+\frac{12 \ell^{2}\left(a^{2}+e^{2}\right)}{\left(\ell^{2}+a^{2}\right)^{2}}\right)\right)}{\left(1+\frac{12 \ell^{2}\left(a^{2}+e^{2}\right)}{\left(\ell^{2}+a^{2}\right)^{2}}\right)^{\frac{3}{2}}}\right)-1\right]^{\frac{1}{2}},
$$

and

$$
Y_{ \pm}=\frac{M}{2 X_{+}} \pm \frac{X_{+}^{2}}{\ell^{2}} \pm \frac{1+\frac{a^{2}}{\ell^{2}}}{2}
$$

For $\Lambda<0$, we get two real roots when $Y_{-}>0$, which are physically acceptable and the remaining two are imaginary, when $Y_{-}<0$ all the roots are imaginary which are not physically acceptable but when $Y_{-}=0$ the first two roots coincide and the remaining roots are imaginary. The typical plot showing the positions of horizons for $\Lambda<0$ are shown in the Figure(1). We emphasize the general properties of

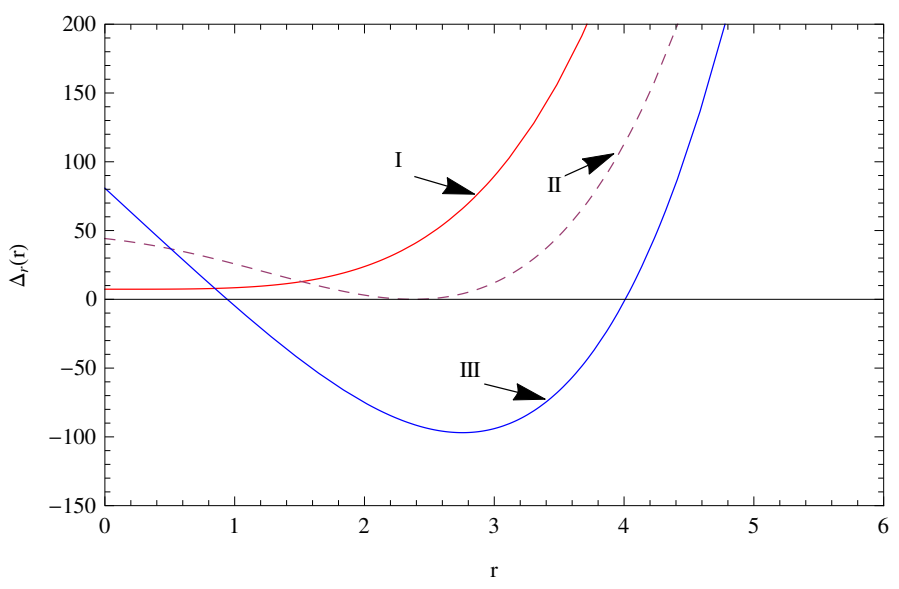

Figure 1. Graphics showing the positions of event horizons as solutions of $\Delta_{r}=0$ for the case $\Lambda<0$. The curve I shows naked singularity for the values of $e=.1, a=.4, \ell^{2}=.007$ and $M=889$, II for extremal event horizon at the values of $e=.1, a=.4, \ell^{2}=.7$ and $M=79$ and the curve line III for two well defined event horizons of the black hole with the values of $e=.1, a=.7, \ell^{2}=.7$ and $M=89$.

a test particle in the region of the black hole where $0<r<r_{-}$. As analyzed in [15], here we rederive the geodesic equation of motion of massive particle in the Kerr-Newman anti de Sitter spacetimes. The Hamiltonian for the geodesic motion is given by

$$
H\left[p_{i}, x^{j}\right]=\frac{1}{2} g^{i j}\left(p_{i}-\epsilon A_{i}\right)\left(p_{j}-\epsilon A_{j}\right),
$$

where $p_{i}$ is the conjugate momenta to the generalized coordinates $x^{i}$ and $\epsilon$ is the charge of the test particle. Since $H$ does not depend explicitly on an affine parameter $\lambda$, the Hamiltonian is a constant of motion. As such, by using the normalization condition, one finds $H=-\frac{m^{2}}{2}$. If $S=S\left(\lambda, x^{i}\right)$ in which $\lambda$ is the affine parameter, is the action then, the conjugate momenta $p_{i}$ are defined by $p_{i}=\frac{\partial S}{\partial x^{2}}$. The corresponding equation of motion becomes

$$
-\frac{m^{2}}{2}=\frac{g^{i j}}{2}\left(\frac{\partial S}{\partial x^{i}}-\epsilon A_{i}\right)\left(\frac{\partial S}{\partial x^{j}}-\epsilon A_{j}\right) .
$$

Substituting all the contravariant components of the metric into the eqn.(16), we get:

$$
\begin{aligned}
& \frac{I^{2}}{\Delta_{\theta} \sin ^{2} \theta}\left(a E \sin ^{2} \theta-L\right)^{2}-\frac{I^{2}}{\Delta_{r}}\left(\left(r^{2}+a^{2}\right) E-a L+\frac{\epsilon e r}{I^{2}}\right)^{2}+\frac{\Delta_{r}}{\rho^{2}}\left(\frac{\partial S}{\partial r}\right)^{2}+\frac{\Delta_{\theta}}{\rho^{2}}\left(\frac{\partial S}{\partial \theta}\right)^{2} \\
& +r^{2} m^{2}+m^{2} a^{2} \cos ^{2} \theta=0 .
\end{aligned}
$$


Separating the $\theta$ - and $r$ - parts and equating them into a constant, K(say) with opposite sign, we get

$$
\Delta_{r}\left(\frac{\partial S}{\partial r}\right)^{2}-\frac{I^{2}}{\Delta_{r}}\left(\left(r^{2}+a^{2}\right) E-a L+\frac{\epsilon e r}{I^{2}}\right)^{2}+r^{2} m^{2}=-K,
$$

or

$$
\frac{\partial S}{\partial r}= \pm \sqrt{\frac{I^{2}\left(E\left(r^{2}+a^{2}\right)-a L+\frac{\epsilon e r}{I^{2}}\right)^{2}-\Delta_{r}\left(K+m^{2} r^{2}\right)}{\Delta_{r}^{2}}}= \pm \frac{\sqrt{R(r)}}{\Delta_{r}}
$$

where

$$
R(r)=\frac{I^{2}\left(E\left(r^{2}+a^{2}\right)-a L+\frac{\epsilon e r}{I^{2}}\right)^{2}-\Delta_{r}\left(K+m^{2} r^{2}\right)}{\Delta_{r}^{2}}
$$

and

$$
\begin{gathered}
\Delta_{\theta}\left(\frac{\partial S}{\partial \theta}\right)^{2}+\frac{I^{2}}{\Delta_{\theta} \sin ^{2} \theta}\left(a E \sin ^{2} \theta-L\right)^{2}+m^{2} a^{2} \cos ^{2} \theta=K, \\
\frac{\partial S}{\partial \theta}= \pm \sqrt{\frac{\left(K-m^{2} a^{2} \cos ^{2} \theta\right) \Delta_{\theta}-\frac{I^{2}}{\sin ^{2} \theta}\left(a E \sin ^{2} \theta-L\right)^{2}}{\Delta_{\theta}^{2}}}= \pm \frac{\sqrt{V(\theta)}}{\Delta_{\theta}}
\end{gathered}
$$

where

$$
V(\theta)=\left(K-m^{2} a^{2} \cos ^{2} \theta\right) \Delta_{\theta}-\frac{I^{2}}{\sin ^{2} \theta}\left(a E \sin ^{2} \theta-L\right)^{2}
$$

The constant $\mathrm{K}$ is determined such that $V\left(\theta=\frac{\pi}{2}\right)=Q$, Carter's constant. We find $Q=K-I^{2}(a E-L)^{2} \Longrightarrow$ $K=Q+I^{2}(a E-L)^{2}$. We can write the solutions of the above equations as

$$
\begin{aligned}
& S_{r}= \pm \int^{r} \frac{\sqrt{R(r)}}{\Delta r} d r, \\
& S_{\theta}= \pm \int^{\theta} \frac{\sqrt{V(\theta)}}{\Delta \theta} d \theta,
\end{aligned}
$$

where $R(r)$ and $V(\theta)$ are defined above. If there exists a separable solutions with some known constants then, the action can be expressed as

$$
S=-\frac{1}{2} m^{2} \lambda-E t+L \phi+\int^{r} \frac{\sqrt{R(r)}}{\Delta_{r}} d r+\int^{\theta} \frac{\sqrt{V(\theta)}}{\Delta_{\theta}} d \theta .
$$

where we have considered the \pm signs absorbed into the integration because of the necessity of changes of the action only. With this action, the equations of motion of the test particle can be casted in the following differential forms:

$$
\begin{aligned}
& \rho^{2} \frac{\partial r}{\partial \lambda}= \pm \sqrt{R(r)}, \\
& \rho^{2} \frac{\partial \theta}{\partial \lambda}= \pm \sqrt{V(\theta)}, \\
& \rho^{2} \frac{\partial t}{\partial \lambda}=I^{2}\left(r^{2}+a^{2}\right)\left[E\left(r^{2}+a^{2}\right)-a L+\frac{e r \epsilon}{I}\right]-\frac{I^{2} a}{\Delta_{\theta}}\left(a E \sin ^{2} \theta-L\right), \\
& \rho^{2} \frac{\partial \phi}{\partial \lambda}=-\frac{I^{2}}{\Delta_{\theta} \sin ^{2} \theta}\left(a E \sin ^{2} \theta-L\right)+\frac{I^{2} a}{\Delta_{r}}\left[E\left(r^{2}+a^{2}\right)-a L+\frac{e r \epsilon}{I}\right],
\end{aligned}
$$

where

$$
\begin{aligned}
& R(r)=I^{2}\left[E\left(r^{2}+a^{2}\right)-a L+\frac{e r \epsilon}{I^{2}}\right]^{2}-\Delta_{r}\left(Q+I^{2}(a E-L)^{2}+m^{2} r^{2}\right) \\
& V(\theta)=\left(Q+I^{2}(a E-L)^{2}-m^{2} a^{2} \cos ^{2} \theta\right) \Delta_{\theta}-\frac{I^{2}}{\sin ^{2} \theta}\left(a E \sin ^{2} \theta-L\right)^{2}
\end{aligned}
$$


where $E$ and $L$ are constants of motion, $\lambda$ is an affine parameter along the null geodesics and $Q$ is the Carter's constant determining the behavior of the particle's motion in the $\theta$-direction. It is obvious that at the dynamic regions where $\Delta_{r}>0$, there is $R(r)>0$. The radial motion has no turning points there. At the stationary regions, where $\Delta_{r} \geq 0$, the turning points of the radial motion can be determined at the points where $R=0$. The effective potentials $R(r)$ and $V(\theta)$ define the motion of the test particle in $r$ and $\theta$ - directions. Thus, the study of a test particle in the gravitational field of the Kerr-Newman anti de Sitter spacetimes is reduced to the study of motion of the test particle in the effective potentials $R(r)$ and $V(\theta)$. For a spherical orbit for which $\dot{r}=0$, the following conditions are satisfied at some radius $\mathrm{r}$ :

$$
R(r)=0, R(r) \equiv \frac{d R(r)}{d r}=0 .
$$

For the spherical orbit to be stable, the condition $R^{\prime \prime}(r)<0$ must also be satisfied.

\section{Spherical orbits of neutral test particle in the black hole}

\subsection{Orbit of a particle of mass $m=1$}

Using the combined equations of (29), we have the following conditions:

$$
\begin{aligned}
R(r)= & \left(I E a^{2}+I E r^{2}-a I L+\epsilon e r\right)^{2}-\left(Q+m^{2} r^{2}+I^{2}(L-a E)^{2}\right) \\
& \times\left(a^{2}-2 r M+r^{2}+e^{2}-\frac{a^{2} r^{2} \Lambda}{3}-\frac{r^{4} \Lambda}{3}\right)=0 \\
R^{\prime}(r)= & (2 I E r+e \epsilon)\left[-a I L+E I a^{2}+r^{2} I E+e \epsilon r\right]-\left[Q+m^{2} r^{2}+I^{2}(L-a E)^{2}\right]\left(-M+r-\frac{a^{2} r \Lambda}{3}-\frac{2 r^{3} \Lambda}{3}\right) \\
& -m^{2} r\left(a^{2}-2 r M+r^{2}+e^{2}-\frac{a^{2} r^{2} \Lambda}{3}-\frac{r^{4} \Lambda}{3}\right)=0, \\
R^{\prime \prime}(r)= & 2(2 E r I+e \epsilon)^{2}+4 I E\left(-a I L+E I a^{2}+E I r^{2}+e \epsilon r\right)-2\left((I a E-I L)^{2}+Q+m^{2} r^{2}\right)\left(1-\frac{\Lambda a^{2}}{3}-2 \Lambda r^{2}\right) \\
& -8 m^{2} r\left(-M-\frac{\Lambda r a^{2}}{3}+r-\frac{2 r^{3} \Lambda}{3}\right)-2 m^{2}\left(e^{2}-2 M r+a^{2}+r^{2}-\frac{a^{2} r^{2} \Lambda}{3}-\frac{r^{4} \Lambda}{3}\right) .
\end{aligned}
$$

For simplicity, we consider for a neutral $(\epsilon=0)$ test particle with its own mass $m=1$ and the mass $M=1$ of a black hole in the background of KN anti de Sitter spacetimes. In this case, equations (30) reduce to the following forms:

$$
\begin{aligned}
R(r)= & \left(I E a^{2}+I E r^{2}-a I L\right)^{2}-\left(Q+r^{2}+I^{2}(L-a E)^{2}\right)\left(a^{2}-2 r+r^{2}+e^{2}-\frac{a^{2} r^{2} \Lambda}{3}-\frac{r^{4} \Lambda}{3}\right)=(331) \\
R^{\prime}(r)= & 2 I E r\left[-a I L+E I a^{2}+r^{2} I E\right]-\left[Q+r^{2}+I^{2}(L-a E)^{2}\right]\left(-1+r-\frac{a^{2} r \Lambda}{3}-\frac{2 r^{3} \Lambda}{3}\right) \\
& -r\left(a^{2}-2 r+r^{2}+e^{2}-\frac{a^{2} r^{2} \Lambda}{3}-\frac{r^{4} \Lambda}{3}\right)=0 \\
R^{\prime \prime}(r)= & 8 E^{2} r^{2} I^{2}+4 I E\left(-a I L+E I a^{2}+E I r^{2}\right)-2\left((I a E-I L)^{2}+Q+r^{2}\right)\left(1-\frac{\Lambda a^{2}}{3}-2 \Lambda r^{2}\right) \\
& -8 r\left(-1-\frac{\Lambda r a^{2}}{3}+r-\frac{2 r^{3} \Lambda}{3}\right)-2\left(e^{2}-2 r+a^{2}+r^{2}-\frac{a^{2} r^{2} \Lambda}{3}-\frac{r^{4} \Lambda}{3}\right)
\end{aligned}
$$

We introduce a quantity called impact parameter $b$ defined as $b=\frac{L}{E}$, for the neutral massive particle on the spherical orbit. Using the equations.(31)and(32), we find two pairs of solutions for the energy $E$ and impact parameter $b$ as

$$
b_{1,2}=\frac{\left( \pm D_{2} r-a^{2}\left(r-e^{2}-\frac{\Lambda r^{4}}{3}\right)\left[\beta r+Q\left(a^{2}-r\left(r-e^{2}-\frac{\Lambda\left(a^{2}+e^{2}\right)^{2}}{3}\right)\right)\right]\right)}{\left(a\left(r-e^{2}-\frac{r^{4} \Lambda}{3}\right)\left[r\left(\left(\Delta_{r}-a^{2}\right)^{2}-a^{2}\left(r-e^{2}\right)+\frac{\Lambda a^{2} r^{4}}{3}\right)\right]+Q\left(1-r+\frac{2 \Lambda r^{3}}{3}+\frac{\Lambda r a^{2}}{3}\right) a^{2}\right)}
$$




$$
E_{1,2}^{2}=\frac{\left(\begin{array}{c}
\mp 2 D_{2}+\beta_{1} r^{2}+Q a^{2}\left[2\left(r-e^{2}+\frac{\Lambda r^{4}}{3}+\frac{\Lambda r^{2} a^{2}}{3}\right) \Delta_{r}\right. \\
\left.-r^{2}(r-1)^{2}\right]+\frac{6 r^{2} \Lambda\left(a^{2}+2 r^{2}\right)\left(r^{2}-r-\Delta_{r}\right)}{3}-\frac{r^{4} \Lambda^{2}\left(a^{2}+2 r^{2}\right)^{2}}{3}
\end{array}\right)}{\left(\begin{array}{c}
I^{2} r^{4}\left[\left(r-3 r+2 e^{2}\right)^{2}-4 a^{2}\left(r-e^{2}+\frac{e^{2} r^{2} \Lambda}{3}-\frac{2 \Lambda r^{3}}{9}\right.\right. \\
\left.\left.-\frac{\Lambda r^{4}}{6}+\frac{a^{2} r^{4} \Lambda^{2}}{12}+\frac{2 r^{6} \Lambda^{2}}{9}-\frac{a^{2} \Lambda r^{4}}{9}-\frac{2 r^{6} \Lambda}{9}\right)\right]
\end{array}\right)}
$$

where

$$
\begin{gathered}
D_{2}^{2}=\left[a \Delta_{r}\left(r-e^{2}-\frac{\Lambda r^{4}}{3}\right)\right]^{2}\left[r^{4}\left(r-e^{2}-\frac{\Lambda r^{4}}{3}\right)-Q\left(r^{2}-3 r+2 e^{2}-\frac{\Lambda r^{2} a^{2}}{3}\right) r+a^{2} Q^{2}\right] \\
\beta_{1}=\left(-2 a^{2} \Delta_{r}+\left((-1+r) r-2 \Delta_{r}\right) \Delta_{r}^{2}+a^{2}\left((-1+r)^{2} r^{2}-(-1+r) r \Delta_{r}+4 \Delta_{r}^{2}\right)\right) \\
\quad-r^{2}\left(a^{2}+2 r^{2}\right)\left(a^{2}\left(2(-1+r) r-\Delta_{r}\right)+\Delta_{r}^{2}\right) \frac{\Lambda}{3}+\frac{a^{2} r^{6} \Lambda^{2}}{3}\left(a^{2}+2 r^{2}\right)^{2}, \\
\beta_{2}=e^{4}-a^{2}\left(r-e^{2}\right)+2 r e^{2}(r-2)-r^{2}(3 r-4)+\left[\frac{r^{2} \Lambda\left(a^{2}+r^{2}\right)}{3}\right]^{2}+\frac{2 r^{2} \Lambda\left(a^{2}+e^{2}\right)\left(r-e^{2}\right)}{3} .
\end{gathered}
$$

It is easy to verify that, for the first pairs of solution $\left(E_{1}, b_{1}\right)$, stable periodic spherical orbits are realized in the regions of $0<r<r_{-}$in which $r_{-}$is the inner event horizon of the black hole with the condition that will exist only $0<Q<Q_{m}$ in which $Q_{m}$ is the root of the equation $R^{\prime \prime}(r)=0$ and is given by

$$
Q_{m}=-I^{2} L^{2}+\frac{2 a I^{2} E L\left(a^{2}+6 r^{2}\right) \frac{\Lambda}{3}-I^{2} E^{2}\left(a^{2}+6 r^{2}\right)\left(1+\frac{a^{2} \Lambda}{3}\right)}{\left(-1+\frac{a^{2} \Lambda}{3}+2 r^{2} \Lambda\right)}+\frac{e^{2}+a^{2}-\frac{11 a^{2} r^{2} \Lambda}{3}-10 r+11 r^{2}-\frac{29 r^{4} \Lambda}{3}}{2\left(-1+\frac{a^{2} \Lambda}{3}+2 r^{2} \Lambda\right)} .
$$

\subsection{Spherical orbits of photons in the black hole}

The spherical photon orbit corresponds to the limit equivalent to the case when $\epsilon=0, m=0$. Photon orbit is dependent on two parameters: the azimuthal impact parameter $b=L / E$ and the latitudinal (tangential) impact parameter $q=Q / E^{2}$. The stability condition given eqn.(29) in the ultra relativistic limit for very massive particle energy for the spherical orbit is simple and found as

$$
\begin{gathered}
b_{1}=\frac{1}{a\left(1+\frac{2}{3} \Lambda r^{3}+\frac{\Lambda r a^{2}}{3}-r\right)}\left[r\left(r^{2}-3 r+2 e^{2}\right)+a^{2}\left(1+r+\frac{\Lambda r a^{2}}{3}\right)\right], \\
q_{1}=\frac{I^{2} r^{2}}{a^{2}\left(1-r+\frac{2}{3} \Lambda r^{2}+\frac{\Lambda a^{2} r}{3}\right)^{2}}\left[4 a^{2}\left(\frac{a^{2} r^{2} \Lambda}{6}-\frac{a^{2} r^{4} \Lambda^{2}}{9}+r-e^{2}-r^{3} \Lambda+\frac{2}{3} r^{2} e^{2} \Lambda\right)-\left(-3 r+r^{2}+2 e^{2}\right)^{2}\right], \\
b_{2}=\frac{r^{2}+a^{2}}{a}, \\
q_{2}=-\frac{I^{2} r^{4}}{a^{2}} .
\end{gathered}
$$

Here, it is verified that the first pair of impact parameters $\left(b_{1}, q_{1}\right)$, corresponds to stable spherical photon orbits, while the second pair $\left(b_{2}, q_{2}\right)$, to unstable ones in the inner horizon of the black hole but just before the singularity of the black hole.

\subsection{Circular orbits as a relativistic limits of spherical orbits}

The equatorial circular photon orbit reduces from the the spherical orbits. Such circular orbit is equivalent to the case when $\epsilon=0, m=0, Q \rightarrow 0$ in the relativistic limit of the spherical orbits. The stability conditions given by eqn.(29) for orbits give two equations as

$$
R(r)=\left(-a L+\left(a^{2}+r^{2}\right) E\right)^{2}-\left\{\left(1-\frac{1}{3} \Lambda r^{2}\right)\left(r^{2}+a^{2}\right)-2 r+e^{2}\right\}(a E-L)^{2}=0,
$$




$$
\begin{gathered}
R^{\prime}(r)=\left(-a L+\left(a^{2}+r^{2}\right) E\right) 2 E r-\left(r-\frac{2}{3} \Lambda r^{3}-\frac{\Lambda r a^{2}}{3}-1\right)(a E-L)^{2}=0, \\
R^{\prime \prime}(r)=8 E^{2} r^{2} I^{2}+4 I^{2} E\left(-a L+E a^{2}+E r^{2}\right)-I^{2}(a E-L)^{2}\left(2-\frac{2 a^{2} \Lambda}{3}-4 r^{2} \Lambda\right),
\end{gathered}
$$

from which we have a corresponding relation for equatorial circular photon orbits in the relativistic form as

$$
\left(r-3 r+2 e^{2}\right)^{2}=4 a^{2}\left(r-e^{2}+\frac{e^{2} r^{2} \Lambda}{3}-\frac{2 \Lambda r^{3}}{9}-\frac{\Lambda r^{4}}{6}+\frac{a^{2} r^{4} \Lambda^{2}}{12}+\frac{2 r^{6} \Lambda^{2}}{9}-\frac{a^{2} \Lambda r^{4}}{9}-\frac{2 r^{6} \Lambda}{9}\right) .
$$

The photon orbit depends on the latitudinal (tangential) impact parameter $q=Q_{r e l} / E^{2}$. The equation in the ultra relativistic limit gives two possible solutions for impact parameters:

$$
b_{1,2}=\frac{ \pm D_{2} r^{2}+a \beta_{2}}{\left[\left(\Delta_{r}-a^{2}\right)^{2}-a^{2}\left(r-e^{2}\right)+\frac{\Lambda a^{2} r^{4}}{3}\right]},
$$

where

$$
D_{2}^{2}=\Delta_{r}^{2}\left(r-e^{2}-\frac{\Lambda r^{4}}{3}\right)
$$

and

$$
\beta_{2}=e^{4}-a^{2}\left(r-e^{2}\right)+2 r e^{2}(r-2)-r^{2}(3 r-4)+\left[\frac{r^{2} \Lambda\left(a^{2}+r^{2}\right)}{3}\right]^{2}+\frac{2 r^{2} \Lambda\left(a^{2}+e^{2}\right)\left(r-e^{2}\right)}{3} .
$$

We verify that, for the first pair of impact parameters, $\left(b_{1}, q_{1}\right)$, stable periodic equatorial circular orbits are realised in the regions of $0<r<r_{-}$in which $r_{-}$is the inner event horizon of the black hole, while the second pair, $\left(b_{1}, q_{2}\right)$, to unstable ones.

\section{Conclusion}

We have investigated geodesic equations of a test particle in the Kerr-Newman (KN) anti de Sitter spacetimes in light of the Hamiltonian-Jacobi equation of motion. It has been observed that in the internal space-time domain $0<r<r_{-}$where $r_{-}$is the inner horizon of the black hole, there exists spacious R-region where a test particles may trace a spherically stable orbits which are bounded and finally, it is shown that the circular orbit as a relativistic limit of the spherical orbit of the test particle, can exist the stable and bound orbits in the black hole in framework of the anti de sitter spacetimes.

\section{Acknowledgment}

The authors would like to thank the reviewers for good suggestions and comments and thanks to the Inter-University Centre for Astronomy and Astrophysics (IUCAA), Pune for providing hospitality and support during the preparation of the paper. Shanjit Heisnam is grateful to the University Grants Commission for providing him financial assistance in the form of UGC-BSR fellowship.

\section{References}

1. D. B. DeWitt, C., Black Holes Les Astres Occlus. Gordon and Breach Science Publishers, 1973.

2. A. Sen, "Developments in superstring theory," arXiv:hep-ph/9810356, 1998.

3. B. P.J.E.Peebles, "The cosmological constant and dark energy," Reviews of Modern Physics 75, 559, 2003.

4. A. et al., "Supernova search team collaboration," APJ, 2004.

5. S. M. Copeland, E. J. and S. Tsujikawa, "Dynamics of dark energy," Int. J. Mod. Phys. D, 2006.

6. P.J.E.Peebles, "The standard cosmological model," Astro-ph, 1998.

7. S. Chandrasekhar, The Mathematical Theory of Black Holes. Oxford University Press, New York, 1983.

8. Dokuchaev, "Is there any life inside black hole?" Class. Quantum Grav., 2011. 
9. Z. S. J. Bicak and V. Balek, "The motion of charged particles in the field of rotating charged black holes and naked singularities-ii," Bulletin of the Astronomical Institutes of Czechoslovakia, 1989.

10. J. B. V. Balek and Z. Stuchlik, "The motion of the charged particles in the field of rotating charged black holes and naked singularities -ii," Bulletin of the Astronomical Institutes of Czechoslovakia, 1989.

11. J. K. E. Hackmann, V. Kagramanova and C. Lammerzahl, "Analytical solution of the geodesics equation in kerr-(anti)de sitter space-times," Physical Review D, 2010.

12. K. Y. S. Shanjit Heisnam, Irom Ablu Meitei, "Motion of a test particle in the kerr newman de/anti de sitter spacetime," Int. Journal of Astronomy and Astrophysics, 2014.

13. Martin, "Spectral geometry of cosmological and event horizons for kerr-newman de sitter metrics," math-ph, 2011.

14. G. C. Holmes, "The use of hyperbolic cosines in solving cubic polynomials," Math. Gazette, 2002.

15. B. Carter, "Global structure of the kerr family of gravitational fields," Phys.Rev., 1968. 